ACTA UNIVERSITATIS LODZIENSIS

Folia Litteraria Romanica 16, 2021

https://doi.org/10.18778/1505-9065.16.18

Alicja Kacprzak

Université de Łódź

(iD) https://orcid.org/0000-0002-3113-8534

alicja.kacprzak@uni.lodz.pl

Andrzej Napieralski

Université de Łódź

(iD) https://orcid.org/0000-0002-9811-924X

andrzej.napieralski@uni.lodz.pl

\title{
Histoire d'un amour et d'une haine inconditionnels : les surnoms et les sobriquets des équipes de football
}

\begin{abstract}
RÉSUMÉ
Notre article aborde la question des appellations non officielles des équipes de football, qui tout en existant à côté des appellations officielles de celles-ci, témoignent souvent des sentiments forts de leurs supporters et de leurs adversaires. Par exemple, à côté de la dénomination officielle, le Futbol Club Barcelona est souvent désigné par les médias au moyen des noms métonymiques évoquant les couleurs du club, Blaugranes ou Azulgranas, mais aussi par un nom plus affectif, Barça. Or, ce même club se voit parfois attribuer une dénomination dépréciative, culés, venant de cul 'derrière' en catalan, ou encore polacos 'Polonais' (nom péjoratif par lequel les Espagnols désignent les Catalans). Notre analyse des formes, du contenu et des rôles des appellations officieuses d'un certain nombre d'équipes de football (françaises, espagnoles et polonaises) permet de proposer une nette distinction entre les surnoms et les sobriquets, deux catégories de chrématonymes bien distinctes.
\end{abstract}

MOTS-CLÉS - onomastique, chrématonyme, surnom, sobriquet, fonctions du langage, football, amour

\section{A Story of an Unconditional Love and Hate: the Nicknames of Football Teams}

\section{SUMMARY}

Our article is a lexical analysis that concerns the world of football and speaks about unofficial names for football teams, which exist alongside their official names and often reflect the strong feelings of their supporters and their opponents. For example, beside the official name, Futbol Club Barcelona the media use metonymic names evoking the colors of the club like Blaugranes or Azulgranas, but they also use a more emotional name - Barça. However, this same club is sometimes given 
a pejorative name - culés, coming from cul 'behind' in Catalan, or polacos 'Poles' (the pejorative name by which the Spanish designate the Catalans). Our analysis of the forms, content and roles of the unofficial names of some (French, Spanish and Polish) football teams allows us to propose a clear distinction between two types of nicknames: surnoms and sobriquets, two very distinct categories of chrematonyms.

KEYWORDS - onomastics, chrematonym, language functions, nickname, football, love

\section{Introduction}

Parmi différentes facettes de l'amour, la passion que les supporters de football vouent à leur équipe préférée constitue un cas particulier. Cet amour inconditionnel, proche souvent d'une dévotion (Dominik Antonowicz et Łukasz Wrzesiński, 2009, évoquent à ce propos une « religion invisible », terme employé d'abord par Thomas Luckmann, 1987), est manifesté soit de façon iconique au moyen des banderoles déployées pendant les matchs ou encore au moyen des écharpes de supporters (Kacprzak, Napieralski, 2015), soit de façon verbale au moyen des chants de stade et des slogans, soit des deux façons à la fois, car les banderoles et les écharpes peuvent comporter aussi bien des textes que des symboles. Il ne faut pas oublier, cependant, que l'amour pour son équipe s'accompagne souvent de la haine pour d'autres équipes, elle aussi vivement exprimée, surtout dans le milieu des ultras (l'animosité entre les ultras des clubs de Łódź est présentée plus largement notamment dans Napieralski, $2014: 142$ ).

Il est bien connu que de nombreux instruments langagiers servent à exprimer des sentiments. Catherine Kerbrat-Orecchioni énumère à ce propos, à côté des moyens syntaxiques tels que l'emphase, la rupture de construction et l'inversion, aussi des moyens morphologiques, dont la suffixation diminutive ou augmentative, et surtout des moyens lexicaux, parmi lesquels on trouve essentiellement les exclamations et interjections, les expressions figées, les mots tendres et les injures (Kerbrat-Orecchioni, 2000 : 33). Il nous semble utile d'ajouter à cette liste certains noms propres dont on peut constater un usage émotif. C'est le cas notamment des surnoms et des sobriquets, dont le caractère non officiel admet facilement une coloration émotionnelle. Leur fonctionnement englobe deux aspects : d'un côté, ils marquent l'émotion du locuteur, de l'autre ils servent à présenter l'entité dénommée sous un jour mélioratif ou péjoratif.

C'est ainsi que les appellations non officielles des équipes de football, qui accompagnent leurs noms officiels, témoignent des sentiments forts de leurs supporters, mais aussi de leurs adversaires. En effet, la dénomination officielle le Futbol Club Barcelona est doublée par des noms métonymiques venant des couleurs du club, Blaugranes en catalan et Azulgranas en espagnol, souvent employés par les médias, ainsi que par une appellation ayant un caractère plus affectif: Barça. Or ce même club est parfois désigné aussi par des noms dépréciatifs, 
notamment culés, venant de cul 'derrière' en catalan, ou encore polacos 'Polonais' (nom péjoratif par lequel les Espagnols désignent des Catalans en général). Dans notre étude qui se veut onomastique, nous nous proposons d'analyser les formes et les valeurs sémantiques des noms non officiels d'un certain nombre d'équipes de football, surtout espagnoles, françaises et polonaises, ceci afin de découvrir les rôles qu'ils remplissent dans le discours. Nous avançons l'hypothèse que ceuxci sont en liaison avec le type d'appellations officieuses parmi lesquelles nous postulons de faire une nette distinction entre les surnoms et les sobriquets, souvent confondus.

\section{Pour une catégorisation du surnom et du sobriquet}

$\mathrm{Vu}$ la complexité de l'onomastique en tant que branche de la linguistique, il nous semble nécessaire de préciser la place qu'est susceptible d'y occuper l'étude des surnoms et des sobriquets de clubs sportifs. En effet, il convient d'envisager cette question selon deux points de vue : d'abord en précisant le champ de l'onomastique dont relèvent les noms de groupes sportifs, ensuite en situant le surnom et le sobriquet parmi d'autres noms propres s'appliquant à des humains, en tant qu'appellations spécifiques résultant de leur caractère informel.

Ainsi, afin de catégoriser les noms d'équipes de football au sein des noms propres, rappelons les trois classes fondamentales de ces derniers : les bionymes (dont les anthroponymes et les zoonymes), les toponymes (avec un vaste éventail de noms dits géographiques) et les chrématonymes, à savoir les noms « qui dénotent différents realia qui résultent d'une activité culturelle de l'homme », comme l'indique Artur Gałkowski (2008 : 9). La notion d'activité culturelle doit être envisagée de façon très large, car il n'est pas seulement question d'objets matériels produits par l'homme, mais aussi d'entités non matérielles de création humaine, ayant une existence sociale, qui doivent être de ce fait officiellement dénommées. Dans son ouvrage de référence consacré à la chrématonymie, Gałkowski propose une typologie de l'ensemble de chrématonymes, en en distinguant trois classes : les noms de marques (comportant essentiellement les noms de firmes), les noms d'entités culturelles (comportant les noms de fêtes, de festivals, de concours) et les noms de groupements humains. Ces derniers (comportant les noms d'organisations variées, de groupes religieux et de partis) malgré les différences qui concernent l'essence de leur existence, ont en commun d'être rassemblés chacun autour d'une activité et d'un but partagé, que ceux-ci soient sociaux, idéologiques ou politiques (Gałkowski, 2008). C'est au sein des noms de groupements qu'il nous semble licite de classer les noms de clubs de football, même si de nos jours, avec la commercialisation du sport, ils se rapprochent souvent des noms de marques.

Pour ce qui est du surnom, traditionnellement classéparmi d'autres anthroponymes (qu'il suffise de rappeler le nom informel de Coccinelle pour la Volkswagen type 1), 
il se place à côté du prénom, du nom de famille patronyme ou matronyme, du nom patronymique (employé entre le prénom et le nom de famille, par exemple en Russie), du postnom (qui suit le nom de famille, notamment dans la culture bantoue), de l'allonyme et du pseudonyme, dont il se distingue par un ensemble de traits spécifiques. Ainsi, le prénom, le nom de famille, le nom patronymique et le postnom, sont certifiés dans les documents de l'individu en tant que ses dénominations valables dès la naissance, dont il a hérité ou qui lui ont été attribuées par ses parents. Leur fonction est avant tout de reconnaître officiellement l'existence sociale de l'individu : notons à ce propos différents cas d'interventionnisme administratif et/ou religieux en ce qui concerne la structure des dénominations complètes de l'individu dans une communauté (le choix des éléments et leur ordre, le fait d'interdire ou de favoriser certains prénoms etc.). L'allonyme et le pseudonyme sont par contre choisis délibérément par l'individu lui-même à un moment de sa vie, afin de remplir une fonction culturelle ou bien cryptique : comme tels, ils ne relèvent pas de l'administration, même s'ils sont connus voire célèbres et fonctionnent très largement dans l'espace public.

Le surnom, qui ne fait pas partie des appellations officielles, n'est pas cependant choisi par l'individu qui le porte, mais lui est attribué par les autres. Qui plus est, il semble toujours marqué par une sorte d'évaluation affective qui accompagne son attribution. En effet, comme l'indique le $T L F i$, il met en relief « une particularité physique, une qualité morale ou une action d'éclat (Pépin le Bref, The Iron Lady, Soliman le Magnifique)», ou bien, il constitue une appellation familière ou pittoresque (Bambi pour Tony Blair, Tonton pour François Mitterrand, Babar pour Raymond Barre), auquel cas il s'agit d'un sobriquet ${ }^{1}$. Cette dernière définition, à laquelle nous souscrivons, permet par conséquent de distinguer la catégorie du surnom, englobant les appellations informelles, mais gardant un ton globalement sérieux et stylistiquement neutre, de celle du sobriquet, présentant un ton de dérision et relevant de la variante non standard de la langue. Ainsi, en revenant à l'exemple cité au début de cet article, Blaugranes et Azulgranas, relevant du registre standard de la langue, constitueraient des surnoms, alors que Barça, culés et polacos, relevant du familier ou populaire, constitueraient des sobriquets. Cette différence de style accompagne une opposition notable en ce qui concerne les valeurs véhiculées par les deux types d'appellations. En effet, les deux surnoms énumérés qui renvoient aux couleurs symboliques de l'équipe de Barcelone ont un caractère noble et sous-tendent une " idée honorable » (ce qui est indiqué comme l'une des propriétés du surnom selon l'Encyclopédie, ou Dictionnaire raisonné des sciences, des arts et des métiers, $1775: 252)$. Au contraire, les sobriquets culés et polacos non seulement comportent une charge axiologique négative, mais apportent en plus une nuance de déconsidération ; c'est le cas aussi du sobriquet Barça qui est marqué, malgré sa charge axiologique positive, par une nuance de diminution du fait de son caractère familier.

\footnotetext{
${ }^{1}$ http://stella.atilf.fr/Dendien/scripts/tlfiv5/advanced.exe?8;s=2623775070, consulté le 24 octobre 2019.
} 


\section{Les surnoms et les sobriquets des équipes de football : vue contrastive}

Les différences stylistiques qui séparent les deux classes se manifestent d'un côté au niveau formel et de l'autre au niveau sémantique des unités. Sera abordée ci-dessous dans une perspective contrastive l'analyse des formes et du contenu sémantique des surnoms et des sobriquets, afin de découvrir des régularités éventuelles auxquelles obéissent les deux catégories mentionnées.

\subsection{Les formes des surnoms et des sobriquets}

Une opposition assez nette qui sépare les surnoms des sobriquets suit celle qui marque les limites entre les procédés lexicogéniques que l'on considère comme relevant de la variante standard et ceux qui sont vus comme appartenant à la variante substandard.

2.1.1. Les formes des surnoms sont constituées surtout par des mots dérivés et des mots composés, on y ajoute aussi des mots simples. Dans le cas des dérivés, il est question des gentilés formés sur la base d'un nom indiquant le lieu d'origine d'une équipe, avec cette précision cependant que le plus souvent ce sont des noms historiques. Prenons comme exemple le surnom Los Numantinos attribué au club CD Numancia, résidant en fait à Soria, une petite ville située dans la communauté autonome de Castille-et-León. L'appellation officielle du club évoque le nom d'une ville antique celtibère (située près de Soria) connue pour sa résistance à la conquête romaine, entre 153 av. J.-C. et 133 av. J.-C. La dénomination Los Numantinos constituerait donc un ethnonyme collectif dérivé du nom de ville Numantia, à valeur symbolique évidente. À son tour, le surnom Les Phocéens est utilisé pour parler du club l'Olympique de Marseille. Cette appellation provient indirectement du nom de Phocée, ancienne cité grecque située sur la côte de la mer Égée. Dans son histoire, Phocée est devenue une cité-mère de la colonisation grecque en Méditerranée occidentale : l'actuelle Marseille a été fondée en 600 av. J.-C. par les Phocéens, d'où son appellation de « cité phocéenne ». Dans les deux cas, les ethnonymes pour ainsi dire historiques sont d'une manière évidente plus expressifs que des gentilés dérivés des noms actuels des deux villes, à savoir los Sorianos et les Marseillais.

Les noms composés utilisés comme surnoms des équipes de football comportent le plus souvent les noms des couleurs symboliques des clubs, comme c'est le cas de l'appellation Les Tricolores de l'équipe de France, Los Blanquinegros 'les blanc et noir' se rapportant à Valencia Club de Fútbol, ou encore Biato-zieloni 'les blanc et vert' du club polonais Lechia Gdańsk.

Dans le cas des noms simples utilisés comme surnoms, il est question d'habitude d'évoquer les symboles relatifs à différents clubs, comme Los Leones 'les lions' pour l'Athletic de Bilbao, ou bien Orly 'les aigles', pour l'équipe nationale polonaise. 
2.1.2. Les formes des sobriquets, comme nous venons de le signaler ci-dessus, relèvent surtout des procédés formateurs substandards, comme la troncation avec ses variantes, ainsi que la mot-valisation. Ainsi, les abréviations parmi les sobriquets des équipes de football sont légion, ce qui est dû sans doute au besoin de s'exprimer d'une manière brève et expressive qui caractérise le style non standard. Comme exemple, citons l'appellation Guada, apocope du nom de la ville dans le nom officiel du club CD Guadalajara. Le sobriquet Jaga est constitué par une forme abrégée du nom Jagiellonia Białystok, celui de Tef résulte de la contraction du nom plein de Toulouse FC, acronymisé en le Téfécé.

La siglaison constitue aussi un procédé exploité pour désigner de façon brève des clubs sportifs; souvent les sigles en question prennent la forme des acronymes qui est gardée même à l'écrit, comme dans le cas cité plus haut du Téfécé, contraction du nom Toulouse FC. Il en va de même en ce qui concerne l'unité Gieksa, acronyme modifié à la forme du féminin de l'appellation officielle du club GKS Katowice. Les sigles constituent aussi les bases de la suffixation au moyen de -iste, comme dans le cas des dénominations les Asémistes et les Scoïstes, créées respectivement sur le nom du club AS Monaco et sur la deuxième partie du nom de l'équipe Angers SCO.

Les mots-valises, enfin, apparaissent aussi comme formes de sobriquets désignant les équipes de football. Citons comme exemple l'unité Kuchenkorz rassemblant les termes préexistants kuchenka 'cuisinière' et kolejorz 'cheminot'. Ce dernier constituait un sobriquet plus ancien de l'équipe de Lech Poznań, vu que le club a été lié pendant six décennies à l'entreprise nationale polonaise de chemins de fer. Depuis une quinzaine d'années, le club est mutualisé avec un autre, lié à un fabricant d'électroménager polonais, d'où l'allusion à la cuisinière, premier élément contenu dans le mot valise.

\subsection{Le contenu sémantique des surnoms et des sobriquets}

Le contenu sémantique des surnoms des équipes européennes relève de plusieurs groupes de sens. Les couleurs qui constituent des symboles, que ce soit à l'échelle nationale, régionale ou urbaine, sont un repère auquel renvoient le plus souvent les surnoms de différentes équipes européennes. Ainsi, le surnom Oranje 'Les Orange' de l'équipe des Pays-Bas provient de la couleur orange du premier drapeau néerlandais, né de la révolte des $16^{\mathrm{e}}$ et $17^{\mathrm{e}}$ siècles contre la domination espagnole. Dans le cas de l'appellation Azzurri 'Les Bleus' de l'équipe d'Italie, il est aussi question d'une couleur à valeur historique, le bleu et le blanc étant les couleurs de la Maison de Savoie. Dans nombre de cas, le surnom comporte plusieurs noms de couleurs, celles qui apparaissent sur le drapeau, comme dans Die Rot-Weiss-Roten 'Les Rouges-Blancs-Rouges', pour désigner l'équipe de l'Autriche, ou dans Blågult 'Les Bleus et Jaunes', celle de la Suède. Il arrive 
par ailleurs que les teintes ne soient pas spécifiées, comme dans le surnom Les Tricolores, donné à l'équipe nationale française, ou bien qu'on les indique de manière imagée, comme dans l'appellation Kockasti 'Les Damiers' de l'équipe nationale croate où le damier comportant les carreaux rouges et blancs symbolise les 25 provinces du pays. L'adjectif de couleur peut aussi accompagner un nom véhiculant un autre symbolisme particulier, comme dans De rode Duivels (néerl.) et Les Diables rouges (fr.), les surnoms qui font référence à l'équipe belge. Il en va de même pour The Boys in Green 'Les Gars en vert', le surnom de l'équipe d'Irlande, ou encore Białe Orty 'Les Aigles blancs', le surnom de l'équipe nationale polonaise.

Les animaux dont la valeur symbolique est marquée tout particulièrement constituent un autre repère, assez fréquemment exploité dans les surnoms d'équipes européennes ${ }^{2}$. Il est à souligner que les animaux en question, réels ou fictifs, sont surtout des symboles de force, parfois de férocité. Ainsi le symbolisme du mot dragon est mis à profit entre autres dans le cas de Zmajceki, surnom de l'équipe slovène, Zmajevi, surnom de l'équipe de la Bosnie et de l'Herzégovine, ainsi que dans The Dragons, Y Dreigiau (en gallois), surnom de l'équipe du Pays de Galles. Le lion, un autre animal connu pour sa puissance, apparait par exemple dans le surnom de l'équipe bulgare, Лъвовете. Parmi les oiseaux c'est surtout l'aigle et le faucon, symbolisant force et prestige, qui trouvent leur place dans de nombreux surnoms, comme Białe Orty 'Les Aigles blancs', pour l'équipe de Pologne ou Hrabri Sokoli 'Les Faucons courageux' pour celle du Monténégro. Notons cependant aussi des symbolismes plus rares dans le domaine de sport, par exemple le grand-duc dont le nom apparaît dans le surnom de l'équipe finlandaise, Huuhkajat 'Les Grands-Ducs'. Cette appellation est due à un match à domicile Finlande-Belgique en 2007, pendant lequel un grand-duc s'est posé sur la barre transversale d'un but, alors que le score était de 0-0. Le match a été interrompu, mais la Finlande a finalement gagné le match et c'est ainsi que l'oiseau est devenu emblématique pour cette équipe. Les traits de caractère, enfin, relèvent des valeurs auxquelles font directement appel les dénominations de quelques équipes de football en Europe. Il est question surtout de l'énergie et de la vaillance des joueurs, comme dans le cas des surnoms La Furia (roja) 'La Furie' pour l'équipe d'Espagne, Vatreni 'Les Ardents' de l'équipe de Croatie, ainsi que de Danish Dynamite 'Les Dynamites danoises' de l'équipe de Danemark.

Les succès présents ou passés d'équipes nationales apparaissent aussi dans leurs surnoms, ceci sous des formes variées. Ainsi Drillos est un surnom de l'équipe de Norvège, qualifiée pour la Coupe du monde 1994 et 1998 sous l'égide du sélectionneur Egil "Drillo" Olsen. Orty Górskiego 'Les Aigles de Górski'

\footnotetext{
2 Notons une fréquence particulière de noms d'animaux dans les surnoms des équipes africaines, par ex. As Palancas Negras 'Les Antilopes noires' (Angola), Likuena 'Les Crocodiles' (Lesotho), Os Mambas ‘Les Mambas' (Mozambique), Os Tubarões Azuis 'Les Requins bleus' (Cap-Vert) etc.
} 
est un surnom de l'équipe de Pologne, en souvenir du sélectionneur Kazimierz Górski, auteur de succès mémorables dans les années 1970. Mágikus Magyarok 'Les Magyars magiques' constitue un surnom de l'équipe nationale de la Hongrie dans les années cinquante, alors que Das Wunderteam 'L'équipe miracle' est celui de l'équipe nationale d'Autriche entre 1930 et 1933.

Le contenu sémantique des sobriquets attribués à des équipes européennes forme aussi quelques groupes de sens, qui ne sont pourtant pas identiques à ceux qu'il a été possible d'énumérer pour les surnoms. Les couleurs, qui renvoient cette fois-ci non pas aux drapeaux ou emblèmes, mais aux maillots de joueurs, constituent un repère important des sobriquets qui se réfèrent à leurs motifs souvent par le biais d'un mécanisme tropique. Ainsi le sobriquet Los Merengues 'les meringues' pour l'équipe du Real de Madrid représente par métaphore la couleur blanche de leurs costumes portés lors des matchs à domicile. Ce sobriquet métaphorique peut être considéré sans doute comme un équivalent moins noble du surnom métonymique Los Blancos. La dénomination Los Colchoneros 'les matelas' employée pour désigner le club Atlético de Madrid fait allusion au motif de rayures rouges et blanches de leurs maillots, qui fait penser à celui de matelas anciens. Ce sobriquet fonctionne parallèlement au surnom Los Rojiblancos qui évoque aussi les couleurs des maillots, mais qui n'a pas de caractère railleur. Le motif de rayures jaunes et rouges est exploité aussi dans le sobriquet Pszczółki 'les petites abeilles' du club Jagiellonia Białystok, qui du fait de sa forme diminutive, n'a pas de valeur directement négative.

Les produits emblématiques de la ville d'origine constituent un repère auquel renvoie un grand nombre de sobriquets attribués à différents clubs de football. Ainsi, l'équipe de Stomil Olsztyn est connue comme Oponiarze 'liés aux pneus' en raison de la fabrication des pneus par le sponsor titulaire de ce club. D'ailleurs ce club a obtenu par métonymie un autre nom plus dépréciatif, à savoir Kondoniarze 'les préservatifs'. Un autre exemple concerne le sobriquet Scyzoryki 'les canifs' de l'équipe Korona Kielce, qui fait allusion à une usine produisant des couteaux, située dans cette ville avant la $\mathrm{II}^{\mathrm{e}}$ guerre mondiale ; cette vieille dénomination est ressortie de l'oubli grâce au rappeur Liroy, originaire de Kielce et de son fameux tube du même nom.

La désignation des joueurs de football est parfois motivée par un produit gastronomique local, comme dans le cas de Paprykarze 'goulaches' pour Pogoń Szczecin, ainsi que dans le cas de Les Thoniers pour l'US Concarneau, en raison de la production de thon en conserve dans la région. Parfois c'est un légume cultivé dans une région, comme pour Los Pimentoneros 'vendeurs de piments' pour le Real Murcia, qui sert de base pour la dénomination non officielle de son club de football.

Les objets symboliques de la ville d'origine, donnent lieu aussi à plusieurs appellations officieuses des équipes de football. Ainsi, le sobriquet Medaliki 'médailles miraculeuses' renvoie au club polonais de Raków relevant de de la ville de Częstochowa, connue pour son sanctuaire de Jasna Góra. 
Les façons de parler de la région d'origine sont évoquées par quelques sobriquets de clubs de football. Les joueurs de Valence CF sont appelés Los Ches par allusion à l'interjection che! utilisée tout particulièrement dans cette région. Les footballeurs du Stade Brestois sont appelés les Ty'Zefs, ce qui constitue une composition des mots bretons ty 'maison' et zef 'vent'. En Pologne, des formes lexicales propres aux patois de la région de Silésie se retrouvent dans les sobriquets Szałoty (déformation du mot sałaty 'salades') pour les joueurs de Unia Racibórz ou Szczewa (déformation de ścierwa 'charogne') pour le Górnik Zabrze.

\section{Les rôles des surnoms et des sobriquets}

Les différences formelles et sémantiques qui séparent les surnoms et les sobriquets font que les rôles remplis par ces appellations attribuées à des clubs de football par des tiers ne sont pas identiques non plus, même si les deux catégories relèvent du non standard.

\subsection{Les surnoms}

Les surnoms des équipes de football fonctionnent massivement dans le discours médiatique qui est non seulement leur utilisateur, mais sans doute aussi leur créateur. En effet, il est bien connu que les créations lexicales imagées fourmillent dans la rhétorique des journalistes sportifs (voir par exemple Vanhoudheusden, 2014), ce qui correspond au besoin d'expressivité du langage réunissant les supporters autour des convictions qu'ils sont censés partager. Les surnoms qui, sans exception, sont sémantiquement motivés, font allusion, de façon plus ou moins directe, à ces convictions. Ces dernières tournent essentiellement autour de la valeur construite sur la base de l'identité territoriale et des valeurs liées à des qualités d'importance particulière au sein de la communauté de supporters.

Ainsi un sentiment d'appartenance et d'identification à un territoire revêt une importance particulière du fait de constituer l'un des éléments fondateurs d'inclusion et d'exclusion sociale, constitutif de chaque collectivité, dont celles de communautés de supporters. Dans ce cas, l'idée de territoire est spécifique : en effet, elle correspond à un lieu géographique (ville, quartier, région, pays) d'où provient l'équipe préférée. Pour les fans, le nom de ce lieu désigne de façon métonymique aussi leur club, par conséquent cette appellation détient un pouvoir symbolique, en tissant et en renforçant l'attachement des supporters à leur équipe qui se manifeste au moyen de signes territoriaux, comme les couleurs et les emblèmes des équipes. Les surnoms évoquant ces signes, tels Die Rot-WeissRoten, Бели Орлови ('les aigles blancs') ou encore Los Numantinos, rassemblent les fans des équipes et par le biais des symboles évoqués renforcent leur identité tribale, enracinée dans celle d'appartenance au territoire en question. 
Les surnoms de clubs font allusion aussi à des qualités d'importance particulière pour la communauté de supporters. Il est question notamment de vertus requises dans le milieu comme la puissance physique, le courage et la vaillance. Les dénominations d'équipes telles que The Eagles 'les aigles', Lwy Pótnocy 'les lions du Nord', Vatreni 'Les Ardents', Danish Dynamite 'Les Dynamites danoises' ou La Furia (roja) 'La Furie' (rouge) d'un côté sont censées les pourvoir de ces qualités, alors que de l'autre elles apportent un soutien psychologique aux supporters mêmes qui s'identifient avec les attributs énumérés.

\subsection{Les sobriquets}

Les sobriquets des clubs de football sont beaucoup plus nombreux que les surnoms. Cela n'étonne pas, vu que les équivalents synonymiques sont plus prolifiques dans les registres plus bas de la langue. Du fait du style particulier des sobriquets qui constituent souvent des créations ludiques, les rôles qu'ils remplissent apparaissent aussi comme spécifiques.

\subsubsection{La glorification de son équipe}

En premier lieu, les sobriquets que les supporters attribuent à leur club préféré ont le rôle de le glorifier, souvent de façon hyperbolique. Dans le cas de Los Galácticos 'les galactiques' (Real Madrid) ou Los Celestes 'les célestes' (Celta de Vigo), les appellations renvoient au symbolisme du cosmos ou du ciel connotant l'univers et des forces surnaturelles. Les dénominations telles que Califas 'les khalifs' (Córdoba CF) ou Władcy Miasta Wtókniarzy 'les gouverneurs de la ville de tisserands' (Widzew Łódź), connotent par contre le pouvoir et la domination.

\subsubsection{La mise en dérision de l'adversaire}

Mis à part l'amour et la dévotion pour leur club, les supporters manifestent en général de la haine voire du mépris envers l'équipe adverse. Cela arrive le plus souvent dans le cas des rivalités de nature historique (entre les clubs les plus médaillés d'un championnat), des derbys (entre les clubs de la même ville ou de la même région) ou des confrontations entre le club de la capitale et les clubs d'autres grandes villes. L'un des instruments servant à humilier les adversaires consiste à les mettre en dérision. Une caractéristique neutre au départ est alors mise en relief et ridiculisée. Dans le cas du club de Levante UD, il est question du lieu où est situé le terrain de jeu. Comme dans les années quarante il se trouvait près d'une rivière à grenouilles, le club a été nommé par dérision les Granotas 'les grenouilles'. 
Dans l'emblème du club de Piast Gliwice figure un oiseau rapace, cependant les adversaires de ce club ne le considèrent ni comme un aigle ni comme un faucon, mais y voient un poulet, d'où le sobriquet Kurczaki 'les poulets' attribué à cette équipe. Les Catalans du F.C Barcelone sont traités de Culés ('les culs') en souvenir de l'ancien petit stade de ce club, où les supporters assis sur les bancs supérieurs du stade exposaient de manière involontaire leurs fesses à l'extérieur de l'enceinte des tribunes. Le nom de cette même partie du corps apparaît aussi dans les sobriquets les culs jaunes (US Quevilly) et les culs rouges (Football Club de Rouen), par allusion aux maillots de joueurs.

À la source de la dérision, il y a parfois la jalousie résultant de la richesse d'un club. Le Paris Saint-Germain, après avoir été racheté par le groupe Qatar Sport Investments, fait l'objet de nombreuses moqueries à ce sujet, accumulant des sobriquets comme les Qataris ou le Qatar Saint Germain. Une situation similaire se présente en Espagne où le club de Getafe CF est appelé le Getafe team Dubai.

\subsubsection{L'avilissement de l'adversaire}

Le monde des supporters est un monde machiste dans lequel la loi du plus fort se manifeste tout particulièrement. La langue des ultras reflète cette force qui est pour eux la seule valeur respectable, ce qui s'accompagne de la brutalité et de la grossièreté du langage qu'ils utilisent pour avilir l'adversaire. Ainsi, certains sobriquets ont pour base des mots vulgaires comme kurwy 'putes' et cwele 'homosexuels', le plus souvent adressés au club Legia Warszawa. Dans les deux cas, il est question d'avilir les joueurs masculins en leur attribuant des épithètes relevant de la sphère de la sexualité qui mettent en doute leur virilité. Il en va de même dans le cas de l'utilisation de la forme féminine d'un gentilé pour se référer aux joueurs d'un club, comme pour 'les Marseillaises' (Olympique de Marseille) et 'les parisiennes' (Paris Saint-Germain). On peut supposer que l'utilisation du féminin, connotant la faiblesse, a pour but de montrer sa supériorité sur le club adverse.

Le sobriquet dépréciatif $p s y$ 'les chiens' (dans la langue populaire, il désigne les policiers), attribué à l'équipe de Wisła Kraków par ses adversaires, rend compte de l'histoire de ce club qui, à l'époque communiste, relevait du ministère de l'Intérieur. Leur rival local, le club Cracovia, est dénommé pejsy 'les papillotes', par allusion à son ouverture aux sportifs de tous les milieux, y compris de nationalité juive, dès sa création au début du $20^{\mathrm{e}}$ siècle. D'autres insultes à coloration ethnique sont utilisées comme sobriquets par les supporters des clubs de Łódź qui se traitent les uns les autres de $\dot{z} y d z i$ 'juifs' (voir Napieralski, 2014 : 143). Dans le cas du club GKS Katowice, c'est le sobriquet cygany 'gitans' (notons l'emploi de la forme populaire cygany, au détriment de la forme correcte cyganie).

Les sobriquets mentionnés témoignent d'une grande animosité entre les supporters voire d'une haine qui subsiste malgré l'évolution des mœurs. Ces 
sobriquets, qui sont souvent vulgaires, xénophobes et racistes, ont comme seule fonction de déprécier l'ennemi (le club adverse et ses partisans). Cette énorme hostilité sert de provocation afin que les matchs entre les équipes rivales soient dotés d'un enjeu supplémentaire.

\section{Les surnoms et les sobriquets : catégories distinctes}

L'étude des formes, du contenu sémantique et des rôles que remplissent les appellations officieuses des équipes de football montre que les surnoms et les sobriquets constituent des catégories distinctes de chrématonymes. Il est à souligner cependant que toutes les deux relèvent du même phénomène de la polynomie qui, selon Vaxelaire, résulte de la renomination de la même entité, quelles qu'en soient les raisons (Vaxelaire, 2009 : 307).

La différence principale se résume par le fait que les surnoms des clubs de football sont en général attribués et utilisés par les médias traditionnels afin d'évoquer une équipe de façon imagée et noble, et ils mettent en avant les qualités de l'équipe ainsi que ses points forts. De ce fait, ils relèvent d'un style standard et véhiculent d'habitude une évaluation favorable, méliorative. Les sobriquets constituent par contre des créations dues à des groupes de supporters et d'ultras qui expriment surtout leur haine et mépris envers un club ennemi. Ils fonctionnent ainsi sur les forums des supporters ou dans les commentaires sur Internet ainsi que dans les échanges verbaux des fanatiques de football. Le symbolisme auquel recourent les sobriquets est vulgaire, agressif, parfois grossier. Par conséquent, leur style oscille entre populaire et trivial, parfois même vulgaire.

L'« idée honorable » que véhicule le surnom s'inscrit bien dans l'idée de la glorification du sport et de la rivalité sur le terrain dans l'esprit fair play qui est sans doute favorisé par les médias sportifs. Au contraire, le sobriquet traduit une force émotive primitive qui reflète le côté obscur du sport, successeur de la guerre et du combat.

\section{Conclusion}

$\mathrm{Au}$ terme de cette recherche qui a permis de montrer une nette distinction entre les surnoms et les sobriquets en tant que catégories séparées de chrématonymes, il nous a cependant été possible de constater un point commun qui les relie. Il s'agit en effet d'une caractéristique importante qu'ils partagent, chacun à sa manière. Nous avons vu que le surnom a pour but d'anoblir, le sobriquet, essentiellement, d'avilir. Il en résulte que les deux possèdent une force persuasive qui permet d'influencer les récepteurs potentiels et leur façon de percevoir les équipes dénommées au moyen de ces appellations officieuses. Elles constituent ainsi des noms propres « arguments » 
(Kacprzak, 2007), à savoir ceux qui fonctionnent dans le discours non seulement à titre de leur valeur déictique évidente, consistant à dénommer une entité de manière individuelle, mais aussi pour leurs valeurs symboliques voire magiques. Comme dans le cas de certains prénoms attribués dans des sociétés primitives afin de pourvoir leurs détenteurs de qualités considérées comme requises dans la communauté, les surnoms et les sobriquets servent à exalter ou à déprécier, ne serait-ce que de façon symbolique, la stature de l'entité désignée.

\section{Bibliographie}

ANTONOWICZ, Dominik, WRZESIŃSKI, Łukasz. (2009), « Kibice jako wspólnota niewidzialnej religii », Studia Socjologiczne, n 192 , p. 115-150

GAŁKOWSKI, Artur (2008), Chrematonimy w funkcji kulturowo-użytkowej. Onomastyczne studium porównawcze na materiale polskim, włoskim, francuskim, Łódź, Wydawnictwo Uniwersytetu Łódzkiego

KACPRZAK, Alicja (2007), " Le nom propre a-t-il une force persuasive ? " in Romanica Olomucensia XVIII, Grandeur et décadence de la parole au XXI ème siècle (S. Mica, R. Zamec éds), Olomuc, Univerzita Palackeho, p. 147-154

KACPRZAK, Alicja, NAPIERALSKI, Andrzej (2015), « Culture et valeurs du monde du football : les messages des écharpes des ultras » in Cultures et valeurs (P. Marillaud, R. Gauthier éds), Toulouse, CALS, p. 147-158

KERBRAT-ORECCHIONI, Catherine (2000), «L'analyse des interactions verbales : la notion de "négociations conversationnelles". Défense et illustration », Lalies, n 20, p. 63-141

LUCKMANN, Thomas (1987), The Invisible Religion: The Transformation of Symbols in Industrial Society, New York, Macmillan

NAPIERALSKI, Andrzej (2014), " De la haine au grotesque : la guerre des graffitis à Łódź » in Fonctions identitaires en situation diglossiques argots - dialectes - patois (A. Kacprzak, J-P. Goudaillier éds), Łódź, Wydawnictwo Uniwersytetu Łódzkiego, p. 141-153

VANHOUDHEUSDEN, Romain (2014), « La création lexicale d'origine métaphorique dans le discours journalistique sportif », Lexis, $\mathrm{n}^{\circ}$ 8. (http://journals.openedition.org/lexis/273, consulté le 21 avril 2020)

VAXELAIRE, Jean-Louis (2009), « Lexicologie du nom propre et onomastique », Nouvelle revue d'onomastique, $\mathrm{n}^{\circ}$ 51, p. 301-315

\section{Sitographie}

http://forum.legionisci.com/archive/index.php/t-3502.html, consulté le 24 octobre 2019

http://redlog.pl/2008/03/12/przydomki-druzyn-pilkarskich, consulté le 24 octobre 2019

http://www.mondedufoot.fr/, consulté le 25 octobre 2019

http://www.nastadionie.pl/smierdziele-zabole-inni-kibole-przydomki-polskich-fanatykow/, consulté le 25 octobre 2019

http://www.parlonsfoot.com/archives/2005/03/30/surnoms-des-quipes/, consulté le 22 octobre 2019 http://www.topito.com/top-surnoms-clubs-francais, consulté le 22 octobre 2019

https://angielskieespresso.pl/post/16025/2017/10/18/skad-wziely-sie-przydomki-klubow-premierleague-cz-1/, consulté le 26 octobre 2019

https://angielskieespresso.pl/post/16092/2017/10/19/skad-wziely-sie-przydomki-klubow-premierleague-cz-2/, consulté le 24 octobre 2019 
https://angielskieespresso.pl/post/16171/2017/10/21/bonus-dla-was-najciekawsze-przydomkiw-nizszych-ligach/, consulté le 28 octobre 2019

https://fracademic.com/dic.nsf/frwiki/1584584\#.C2.A0France, consulté le 24 octobre 2019

https://www.20minutes.fr/nantes/1798071-20160302-football-droles-noms-oiseaux-clubs-francais, consulté le 27 octobre 2019

https://www.culture-generale.fr/sports/89-surnoms-des-clubs-de-football-europeens, consulté le 29 octobre 2019

https://www.htlocalization.com/fr/fun-facts/item/382-quels-sont-les-surnoms-des- $\% \mathrm{C} 3 \% \mathrm{~A} 9$ quipes-nationales-de-football, consulté le 17 octobre 2019

https://www.kibice.net/forum/viewtopic.php?f=1\&t=13669\&start=160, consulté le 23 octobre 2019

Alicja Kacprzak - professeure de linguistique et directrice de la section de linguistique à l'Institut d'Études Romanes de l'Université de Łódź. Sa recherche se concentre sur les variantes non standard du lexique et sur la néologie. Auteure de plus de cent articles et ouvrages, elle a publié récemment L'emprunt en question(s) (2019), ainsi que La néologie de l'adjectif en français actuel (2019). Elle dirige le projet PHC POLONIUM « Recent Borrowings in Contemporary French and Polish. A corpus-Based Digital Study ».

Andrzej Jr. Napieralski (UŁ) - maître de conférences depuis 2011. Il est l'auteur d'une trentaine d'articles sur le français non standard, l'analyse du discours du rap et des réseaux sociaux et les néologismes récents. Après avoir publié, le livre La langue du rap en France et en Pologne (2014), il se focalise sur l'analyse du discours des internautes tant du point de vue de la lexicologie (les procédés lexicogéniques, les néologismes et les emprunts) que de l'analyse du discours (les figures). 